\title{
Chamges of gait kinematics in different simulators of reduced gravity
}

Francesca Sylos-Labini ${ }^{1,2}$, Yuri P. Ivanenko ${ }^{1}$, Germana Cappellini ${ }^{1,2}$, Alessandro Portone ${ }^{1}$, Michael J. MacLellan ${ }^{1}$, Francesco Lacquaniti ${ }^{1,2,3}$

${ }^{1}$ Laboratory of Neuromotor Physiology, Santa Lucia Foundation, via Ardeatina 306, 00179 Rome, Italy, ${ }^{2}$ Centre of Space Bio-medicine, University of Rome Tor Vergata, via Montpellier 1, 00133 Rome, Italy, and ${ }^{3}$ Department of Systems Medicine, University of Rome Tor Vergata, via Montpellier 1, 00133 Rome, Italy.

Running title: gait kinematics in reduced gravity

Correspondence to:

Dr Francesca Sylos-Labini

Laboratory of Neuromotor Physiology, IRCCS Fondazione Santa Lucia, 306 via Ardeatina, 00179 Rome, Italy tel ++39 06.51.50.14.75 fax ++3906.51 .50 .14 .82$

e-mail: f.syloslabini@hsantalucia.it 


\begin{abstract}
Gravity reduction affects the energetics and natural speed of walking and running. But, it is less clear how segmental coordination is altered. Various devices have been developed in the past to study locomotion in simulated reduced gravity. However, most of these devices unload only the body center of mass. Here, we reduced the effective gravity acting on the stance and/or swing leg to 0.16g using different simulators. Locomotion under these conditions was associated with a reduction in the foot velocity and significant changes in angular motion. Moreover, when simulated reduced gravity directly affected the swing limb, it resulted in significantly slower swing and longer foot excursions, suggesting an important role of the swing phase dynamics in shaping locomotor patterns.
\end{abstract}

Keywords: gait kinematics; adaptation; reduced gravity; human locomotion 


\section{Introduction}

Investigating human locomotion in a hypogravity environment has received significant attention in view of the biomechanical principles that determine the kinematics of different gaits (Donelan and Kram 1997; Ivanenko et al. 2002; Everett et al. 2009), adaptability of central pattern generation networks to limb loading and clinical implications for gait rehabilitation (Dietz and Duysens 2000; Dominici et al. 2007; White et al. 2008; Selinov et al. 2009; Sylos Labini et al. 2011). Gravity significantly affects the optimal speed and the characteristics of the walk-run transition as shown in theoretical and experimental studies using reduced gravity simulators (Kram et al. 1997; Minetti 2001; Ivanenko et al. 2011), during parabolic flights (Newman 1996; Cavagna et al. 2001) and lunar exploration (Carr and McGee 2009).

On Earth, reduced gravity can be simulated by counterbalancing the body weight with an upward force applied to the trunk of the subject while during space or parabolic flights a subject loading system can be used to simulate gravity (Gosseye et al. 2010). Walking and running are characterized by specific stance limb dynamics (inverted pendulum and bouncing gait, respectively) (Cavagna et al. 1977; Alexander 1989; Saibene and Minetti 2003). All three major lower limb segments (thigh, shank, foot) contribute to the co-variance of angular motion and to appropriate limb axis length and orientation ('telescopic limb’) behavior resulting in a gait-dependent center-ofmass trajectories (Ivanenko et al. 2007). Although the force of gravity acts on both the stance and swing limb (Mochon and McMahon 1980; Kuo 2001; Doke et al. 2005; Doke and Kuo 2007; Raichlen 2008), most of these studies used reduced gravity simulators that unload the body only during the stance phase (Kram et al. 1997; Griffin et al. 1999; Ivanenko et al. 2002; McCrory et al. 2002; Gravano et al. 2011) under the assumption that the swing limb adapts automatically to the modified gait. However, to what extent is this true? This assumption is based on the fact that swing limb adaptation is not purely passive (Marsh et al. 2004; Doke et al. 2005; Doke and Kuo 2007; Ivanenko et al. 2008) and depends also on the contralateral stance limb motion. 
Here, we investigated whether and how the intersegmental coordination and swing limb dynamics adapt to different reduced gravity simulators. Walking and running rely on the limb oscillatory mechanics (Alexander 1989), and one way to change the natural frequency of the pendulum is to modify the level of gravity. To study the effects of reduced gravity on the swing limb, we used a recently developed unloading stepping exoskeleton that allows stepping in simulated reduced gravity by tilting the device relative to the vertical (Ivanenko et al. 2011) and we compared the results with the classical vertical body weight support. While reduced gravity simulators do not fully replicate the actual reduced gravity conditions (the limbs still have weight, and sensory inputs that rely on gravity, such as the otoliths, are still responsive, in contrast with what happens in real weightlessness), nevertheless they allow us to study how the neural control adapts to body unloading and to net force vectors acting in different directions. The data were collected from subjects walking and running on a treadmill at $1 \mathrm{~g}$ (where $\mathrm{g}$ is the gravitational acceleration on Earth) and at the simulated $0.16 \mathrm{~g}$ gravity level similar to what they would experience on the Moon.

\section{Methods}

\section{Participants}

Participants were seven healthy volunteers (age range between 25 and 45 yrs, four males and three females, body mass $73 \pm 9 \mathrm{~kg}$ [mean $\pm \mathrm{SD}$ ], height $1.77 \pm 0.07 \mathrm{~m}$, mean leg length $0.83 \pm 0.05 \mathrm{~m}$ ). None of the subjects had any history of neurological or orthopedic disease. Informed consent was obtained from all participants. The studies conformed to the Declaration of Helsinki, and informed consent was obtained from all participants according to the procedures of the Ethics Committee of the Santa Lucia Foundation. 


\section{Reduced gravity simulators}

To simulate reduced gravity, we used two different apparatuses: a vertical body weight unloading system and a tilting body weight unloading system (Fig. 1). The detailed description of these systems has been provided previously (Ivanenko et al. 2002; 2011; Gravano et al. 2011). Vertical body weight support (Fig. 1A, left panel) was obtained by supporting the subjects in a harness connected to a pneumatic device that applied a controlled upward force independent of the position of the center of body mass, thus simulating a reduced-gravity environment. As a result, each supporting limb experienced a simulated reduction of gravity proportional to the applied force, while the swinging limb experienced 1g. Two different body weight support (BWS) levels were used: 16 and $0 \%$ of body weight (normal $1 \mathrm{~g}$ condition). The experiments were carried out on a treadmill (EN-Mill 3446.527, Bonte Zwolle BV, The Netherlands).

The tilting body weight support system (Fig. 1A, right panel) was constructed to more realistically simulate the effects of gravity changes on both the stance and swing leg (Italian patent \#Rm2007A000489). The subjects lay on their right side with both legs suspended in the exoskeleton, allowing low-friction joint rotation. The length of the telescopic thigh segment of the exoskeleton was adjusted according to the subject's thigh length, and the leg was attached to the exoskeleton so as to provide the best alignment of the axes of rotation of the hip and knee joints with those of the exoskeleton. The foot segment remained unrestrained in air. Tilted BWS more realistically simulates the force component normal to the treadmill surface (Fig. 1B middle panel) acting on both the center-of-body-mass (COM) and the swinging limbs that would be observed in real weightlessness. However, this system prevents arm oscillations, adds inertia (15 kg chassis and $3 \mathrm{~kg}$ exoskeleton), and somewhat limits trunk movements in the anterior-posterior direction. The construction of the tilting BWS system is based on the idea of neutralizing the component $(\mathrm{mg} \cdot \cos (\alpha)$, where $\alpha$ is the angle of inclination and $\mathrm{m}$ is the body mass) of the gravity force normal to the lying surface (Bogdanov et al. 1971; Hansen 1995), while the component $(\mathrm{mg} \bullet \sin (\alpha))$ of the 
gravity force acting on the body and swinging limbs in the sagittal plane is reduced in relation to the tilt angle. The subject stepped on the treadmill that was tilted to the same angle. The apparatus permitted low-friction up-and-down (relative to the treadmill) sliding of the supporting chassis over two parallel tracks formed by a steel beam (Ivanenko et al. 2011).

In addition, a gravity replacement load was employed using the pneumatic cylinder in order to increase limb loading during stance (Fig. 1B, left panel). This option simulated the effect of gravity only during the stance phase while the subject lay in the horizontal position. The amount of the loading force was chosen to correspond to $16 \%$ of the subject's weight, so that it could be compared with the same unloading level using other reduced gravity simulation techniques.

\section{Experimental protocols}

The subjects participated in two separate sessions. In one session, they walked on a treadmill using the vertical body weight support system (including normal 0\% BWS or 1g condition). In another session, they walked on the treadmill using the gravity replacement load and tilting BWS system. The following two main conditions were studied for all simulation techniques (Table 1): walking at a constant speed $2 \mathrm{~km} / \mathrm{h}$, and running at a constant speed $5 \mathrm{~km} / \mathrm{h}$. Walking at $2 \mathrm{~km} / \mathrm{h}$ at 0.16 g corresponds approximately to the "optimal dimensionless" speed. This is defined by the Froude number $\mathrm{Fr}=\mathrm{V}^{2} / \mathrm{gL}$, where $\mathrm{V}$ is the forward speed and $\mathrm{L}$ is the length of the extended lower limb. Empirically, optimal walking (when there is the maximum recovery of mechanical energy due to the exchange between gravitational potential energy and forward kinetic energy) occurs at Fr 0.25 (cf. Minetti 2001). In addition, we also recorded walking at $1 \mathrm{~g}$ at a similar dimensionless speed ( $5 \mathrm{~km} / \mathrm{h}, \mathrm{Fr} \sim 0.25)$ and running at a faster speed $12 \mathrm{~km} / \mathrm{h}$. A $\sim 5$ min habituation period was allowed before data collection. Walking kinematics typically adapt rapidly to simulated reduced gravity (Kram et al. 1997). From 10 to 15 strides were recorded for each condition in each subject. 


\section{Data recording}

We recorded kinematic data bilaterally at $100 \mathrm{~Hz}$ by means of the Vicon-612 system (Oxford, UK) with nine video cameras spaced around the walkway. Infrared reflective markers (diameter $1.4 \mathrm{~cm}$ ) were attached on each side of the subject to the skin overlying the following landmarks: greater trochanter (GT), lateral femur epicondyle (LE), lateral malleolus (LM), heel (HE), and fifth metatarso-phalangeal joint (5MP). The spatial accuracy of the system was better than $1 \mathrm{~mm}$ (root mean square). For the tilting BWS system, the GT marker of the right side of the body could not be recorded (since the subject laid on the right side); also, the GT and LE landmarks of the left leg were recorded by attaching the $20 \mathrm{~cm}$ sticks with two markers to the appropriate joint and the GT and LE positions were reconstructed as a midpoint between these two markers.

\section{Data analysis}

We analyzed gait kinematics in the sagittal plane. The kinematic data were low pass filtered at $20 \mathrm{~Hz}$ with a zero-lag Butterworth filter. Gait cycle was defined as the time between two successive foot contacts (FC) by the same leg according to the local minima of the vertical displacement of the HE marker (Ivanenko et al 2008). The timing of the lift-off was determined analogously (when the 5MP marker elevated by $2 \mathrm{~cm}$ ). The main limb axis was defined as GT-LM and corresponds to the angle between the segment projected on the sagittal plane and the vertical or the normal to the treadmill surface (positive in the forward direction, i.e. when the distal marker falls anterior to the proximal one).

The following gait parameters were calculated: stride duration, percentage of the stance and swing phase durations, motion of the joint (ankle, knee, hip) and segment elevation (thigh, shank, foot) angles, longitudinal (parallel to the treadmill long axis) excursion of the foot (5MP) marker and longitudinal velocity of the foot (5MP marker) relative to the hip. For the analysis of gait kinematics during walking at a constant speed, data were time-interpolated over individual gait cycles to fit a normalized 100-point time base and averaged across strides (typically 10-15 strides). 
The phase-plane diagrams of foot movement (plots of toe velocity versus position) were also illustrated to characterize the dynamics of foot motion (Osaki et al. 2007; 2008).

The intersegmental coordination of the thigh, shank, and foot elevation angles in the sagittal plane was evaluated in position space as previously described using the principal component analysis (Borghese et al. 1996; Ivanenko et al. 2007). For each eigenvector of the covariance matrix, the parameters $u_{i t}, u_{i s}$, and $u_{i f}$ correspond to the direction cosines (range: [-1 1]) with the positive semi-axis of the thigh, shank, and foot angular coordinates, respectively. We specifically analyzed and plotted the $\mathrm{u}_{3 \mathrm{t}}$ parameter for different conditions since it reflects changes in the orientation of the covariance plane (Bianchi et al. 1998). The planarity of the trajectories was quantified by the percentage of total variation (PV) accounted for by the first two eigenvectors of the data covariance matrix (for ideal planarity $P V=100 \%$ and the 3rd eigenvalue $=0$ ). In addition, the time course of each elevation angle was expanded in Fourier series, and the percent variance accounted for by the first harmonic (fundamental component) and its phase were computed.

\section{Statistics}

Descriptive statistics included means \pm standard deviation (SD) of the mean. Repeatedmeasures (RM) ANOVA was used to evaluate differences in the gait kinematics when walking or running at a constant speed using different simulation techniques. Five different conditions (Table 1) were compared independently for walking and running: gravity replacement load, tilted BWS system, vertical BWS system, normal locomotion at $1 \mathrm{~g}$ at the same speed (2 km/h for walking and $5 \mathrm{~km} / \mathrm{h}$ for running) and normal locomotion at $1 \mathrm{~g}$ at a similar Froude number $(5 \mathrm{~km} / \mathrm{h}$ for walking and $12 \mathrm{~km} / \mathrm{h}$ for running). Post-hoc tests were performed by using the Tukey’s HSD test to compare the difference in the means of all conditions versus $1 \mathrm{~g}$ conditions. Reported results were considered significant for $\mathrm{p}<0.05$. 


\section{Results}

We compared gait parameters during walking at $2 \mathrm{~km} / \mathrm{h}$ at simulated reduced gravity $(0.16 \mathrm{~g})$ using different techniques (Fig. 1B): horizontal stepping using gravity-replacement load, tilted BWS system and vertical BWS system. In all these apparatuses, the loading component acting normal to the treadmill surface during stance was similar $(0.16 \mathrm{~g})$ while the simulated gravity affected the swing phase quite differently depending on the system.

The results are illustrated in Fig. 2. RM-ANOVA showed significant effects of condition on the cycle duration $(F(4,24)=36.294, \mathrm{p}<0.00001)$, with a significant increment of the cycle duration for the gravity replacement load and tilted BWS conditions compared to the $1 \mathrm{~g}$ condition at $2 \mathrm{~km} / \mathrm{h}$ ( $<<0.05$ for all comparisons, Tukey’s post-hoc tests). Similar results were obtained for longitudinal foot excursion $(\mathrm{F}(4,24)=36.706, \mathrm{p}<0.00001)$ and maximal longitudinal foot velocity during swing $(\mathrm{F}(4,24)=432.66, \mathrm{p}<0.00001)$ with the addition of a significant decrease of the longitudinal foot excursion for the vertical BWS condition $(\mathrm{p}=0.016)$. Moreover the longitudinal foot velocity was significantly lower in all simulated reduced gravity conditions $(\mathrm{p}<0.0002$ for all comparisons). Asterisks in Fig. 2 indicate significant differences from walking at $2 \mathrm{~km} / \mathrm{h}$ at $1 \mathrm{~g}$. Note that body unloading affected almost all parameters depicted in this figure. We also compared walking at $2 \mathrm{~km} / \mathrm{h}$ at reduced gravity with that at $1 \mathrm{~g}$ at a similar Froude number (5 km/h), under the assumption that human walking obeys dynamic similarity in different gravitational environments (Minetti 2001; Raichlen 2008). While the relative swing duration was similar (Fig. 2A), the longitudinal foot excursion was still smaller ( $\mathrm{p}<0.05$ ) for all reduced gravity conditions (Fig. 2B).

There were, however, differences between different simulation techniques. For the gravityreplacement load apparatus, the maximal longitudinal foot velocity was significantly slower, while the cycle duration and longitudinal foot excursion were significantly larger than for the vertical BWS system ( $\mathrm{p}=0.0002, \mathrm{p}=0.0002$ and $\mathrm{p}=0.0001$, respectively, Tukey post-hoc tests, Fig. 2). The foot peak velocity tended to be slightly smaller for the gravity-replacement load than for the tilting 
BWS condition, though the differences were not significant $(\mathrm{p}=0.3885)$ likely because the swing phase was only slightly if any affected in both cases by the component of gravity in the sagittal plane (Fig. 1B). Thus, major differences in the cycle duration, longitudinal foot excursion and maximal foot velocity were observed between the vertical vs. tilting BWS systems. There was also a co-variation between cycle duration and swing-phase duration such that the swing phase as a percentage of the cycle time was relatively constant across the 3 loading conditions ( $\mathrm{p}>0.18$ for all comparisons, Fig. 2A).

Fig. 3A illustrates ensemble averaged angular waveforms and covariance plots of thighshank-foot elevation angles (bottom panels) across all conditions. The range of motion of joint angles (Fig. 3B, upper panel) was significantly affected by condition $(F(4,24)=30.010, p<0.00001$, $\mathrm{F}(4,24)=44.437, \mathrm{p}<0.00001$ and $\mathrm{F}(4,24)=5.7528, \mathrm{p}=0.002$ for hip, knee and ankle, respectively). Again, asterisks denote significant differences relative to the 1 g condition as in Fig. 2B. In addition, Tukey’s post-hoc tests revealed a significantly greater range of motion at the knee joint for the vertical BWS system compared to the gravity replacement load and tilted BWS conditions ( $\mathrm{p}=0.004$ and $\mathrm{p}=0.046$, respectively), associated with the lower amplitude in the hip joint $(\mathrm{p}=0.0008$ and $\mathrm{p}=0.005$, respectively). Also, the amplitude of elevation angles (Fig. 3B, lower panel) significantly changed with condition $(\mathrm{F}(4,24)=33.517, \quad \mathrm{p}<0.00001, \quad \mathrm{~F}(4,24)=85.197, \quad \mathrm{p}<0.00001 \quad$ and $\mathrm{F}(4,24)=34.871, \mathrm{p}<0.00001$ for thigh, shank and foot, respectively). Moreover the first harmonic (Fig. 3C) explained a percent of variance significantly higher in low gravity conditions for shank $(\mathrm{F}(4,24)=21.06, \mathrm{p}<0.00001)$ and foot $(\mathrm{F}(4,24)=42.35, \mathrm{p}<0.00001)$ and a lower phase angle $(F(4,24)=6.750, p=0.000118$ for shank and $F(4,24)=13.401, p<0.00001$ for foot $)$.

Planar covariation of limb segment elevation angles was observed for all experimental conditions (PV $>98 \%$, Fig. 3A,D). The orientation of the covariance plane $\left(\mathrm{u}_{3 t}\right)$ was similar at reduced gravity (Fig. 3D, lower panel). The percentage of the variance accounted for by the first two eigenvectors was affected by the condition $(F(4,24)=51.188, p<0.00001$ and $F(4,24)=54.020$, $\mathrm{p}<0.00001$ for $\mathrm{PV}_{1}$ and $\mathrm{PV}_{2}$, respectively), due to significantly thinner loops (higher $\mathrm{PV}_{1}$ and lower 
$\mathrm{PV}_{2}$ ) for all reduced gravity simulators. For the vertical BWS, $\mathrm{PV}_{2}$ was smaller than for the gravity replacement load and tilted BWS ( $\mathrm{p}=0.007$ and $\mathrm{p}=0.045$, respectively, Tukey post-hoc tests). For 3 subjects, $\mathrm{PV}_{2}$ was even smaller than $2 \%$ resulting in a linear, rather than planar, relationship between the elevation angles.

Figures 4 and 5 illustrate the results of running at reduced and normal gravity conditions. The comparison of the gait parameters and the intersegmental coordination during running at 5 $\mathrm{km} / \mathrm{h}$ at simulated reduced gravity using the three different techniques (Fig. 4,5) showed similar trends in the kinematic parameters to those detected for walking trials (Fig. 2,3). In particular, we found changes in the cycle duration (Fig. 4A), longitudinal foot excursion, peak foot velocity (Fig. 4B), amplitude of angular motion and planar covariation (Fig. 5). The only qualitative difference from walking was an increase in the foot excursion for running in the vertical BWS condition (Fig. 4B) rather than its decrease (Fig. 2B).

\section{Discussion}

This study focused on adaptation of gait kinematics to different laboratory conditions simulating a reduction of gravity. Our findings revealed significant changes in the swing phase characteristics and intersegmental coordination depending on the type of simulator (Fig. 2-5), thus rejecting the hypothesis of an automatic matching of the swing phase to stance limb unloading. Below we discuss both similarities and differences between different techniques to simulate a reduced gravity.

Changes in gait kinematics with body weight unloading

We observed planar covariance of segment elevation angles under all tested conditions, including different gaits and different simulations of gravity reduction (Fig. 3,5). These results 
agree with our previous findings when participants walked at varying speeds during increasing vertical body weight support (Ivanenko et al. 2002). Such patterns have been seen in numerous forms of locomotion (Borghese et al. 1996; Cheron et al. 2001; Courtine and Schieppati 2004; Courtine et al. 2005; Ivanenko et al. 2007; Noble and Prentice 2008; Hallemans and Aerts 2009; Funato et al. 2010; Leurs et al. 2012; Ogihara et al. 2012), and indicate an underlying locomotor template for the control of segment elevation angles during gait (Lacquaniti et al. 1999; 2002). One of the proposed interpretations of the phase shifts in the elevation angular waveforms (Fig. 3C, 5C) is that the central nervous system adapts to changes induced by reduced gravity by keeping the constraint of planar covariation and tuning the amplitude and the phase shifts of fundamental harmonics of elevation angles (Lacquaniti et al. 2002; Barliya et al. 2009; MacLellan et al. 2010). BWS evoked minor changes in the covariance plane orientation (Fig. 3D, 5D), however, it consistently affected the width of the covariance loop (it became thinner, see significant decrements in the $\mathrm{PV}_{2}$ parameter, Fig. 3D,5D). The range of joint angular motion also changed at reduced gravity during both walking and running (Fig. 3B, 5B). In particular, the amplitude of proximal (thigh) segment motion was significantly smaller than at $1 \mathrm{~g}$ for all three hypogravity simulation techniques.

It is generally assumed that the stance limb mechanics determines the inverted pendulum mechanism of walking (Magaria 1976; Alexander 1989; Cavagna et al. 2000; Saibene and Minetti 2003; Kuo et al. 2005; Cappellini et al. 2010). The gravity level sets limits to the speed of walking, related to the ratio between centrifugal force and gravitational force acting at the COM. According to the principle of dynamic similarity, geometrically similar bodies that rely on pendulum-like mechanics have similar gait dynamics at the same Froude number, which is inversely proportional to gravity. Indeed, the optimal walking speed and the speed of gait transitions decrease monotonically with body unloading consistent with the predictions of dynamic similarity (Kram et al. 1997; Minetti 2001; Sylos Labini et al. 2011). Additional inertia of the tilted chassis ( 20\% of subjects' mean body mass) or that of the exoskeleton (1.5 kg for each leg) could affect metabolic 
rate or gait kinetics (Chang et al. 2000; Browning et al. 2007), although their influences on the speed and abruptness of gait transitions are minor (Ivanenko et al. 2011). During locomotion in humans and other animals, changes in stepping rate typically result from a change in the duration of the extensor (stance) phase, whereas the flexor (swing) phase duration remains relatively constant (Juvin et al. 2007; Sylos Labini et al. 2011). Nevertheless, the swing phase adapts to reduced gravity. The previous studies on parabolic flights investigating the effect of gravity on walking mechanics demonstrated increments in the swing phase duration (by $29 \%$ at $0.25 \mathrm{~g}$, Roberts 1963, see also De Witt et al., 2010), consistent with our results (Fig. 2). The changes of the swing limb dynamics (maximal longitudinal foot velocity) with BWS bolster the hypothesis that the swing phase depends on limb loading.

In sum, many parameters, such as EMG activity (Sylos Labini et al. 2011), preferred walkrun transition speed (Ivanenko et al. 2011), maximal longitudinal foot velocity (Fig. 2B,4B), amplitude of proximal segment motion (Fig. 3B,5B) and shape of the covariance loop (Fig. 3,5), change in parallel for all BWS systems and in actual reduced gravity (Roberts, 1963; Cavagna et al., 2001; Carr and McGee, 2009; De Witt et al., 2010), thus validating the usage of such systems for simulating a reduced gravity in the laboratory.

\section{Differences in gait kinematics using different simulators of reduced gravity}

Despite similarities in approximating reduced gravity, there were nevertheless essential differences between different simulation approaches. The variables that showed the greatest differences between reduced gravity systems were maximal longitudinal foot velocity and longitudinal foot excursion (Fig. 2,4). Maximal longitudinal foot velocity was greatest in the vertical body weight support condition and decreased in the tilted and gravity replacement load conditions. In addition, these values were much lower than at 1 g. These changes were accompanied by lower longitudinal foot excursion in the vertical body weight support condition when compared to the tilted and gravity load replacement conditions. Donelan and Kram (1997) and Ivanenko et al 
(2002) also showed a slight (yet significant) decrease in longitudinal foot excursion as vertical BWS increases, in line with the present results (Fig. 2B). In contrast, the current results suggest an increase in longitudinal foot excursion with different reduced gravity conditions (Fig. 2B,4B) which may highlight the importance of gravity acting on the legs and the control of swing phase. Although the tilted BWS system matches better the dynamic similarity hypothesis (Donelan and Kram 1997), even for this system the longitudinal foot excursion was slightly but significantly smaller than at $1 \mathrm{~g}$ at equal Froude number (5 km/h, Fr 0.25, Fig. 2B).

Also, a slight increase of the percent of the variance explained by the first principal component may be present when compared to the tilted and gravity replacement load reduced gravity conditions, which results in the width of the covariance loop being thinner. Previous work has related the width of this covariance loop to the phase difference between the thigh and shank elevation angle trajectories (Bianchi et al. 1998; Barliya et al. 2009; MacLellan and McFadyen 2010). Since the amplitudes of the thigh and shank elevation angles are similar in each of the reduced gravity conditions (see Figs 3 and 5), it is likely that this thinner covariance loop is also related to thigh and shank elevation angle trajectory phasing and suggests that during the vertical body weight support condition, the trajectories of these segments are more aligned than in the other reduced gravity conditions.

There are a few studies that directly compared simulated and real microgravity locomotion kinematics. De Witt et al. (2010) reported differences in the joint angle amplitudes and stride time between simulated (horizontal supine suspension) and real weightlessness (parabolic flight) for both walking and running. However, we cannot make a direct comparison of our results with those of the De Witt et al. study, because they attached rubber cords to the legs and this may have modified the swing phase dynamics and the kinematic parameters. On the other hand, the results on running obtained using 'vertical' BWS (see Fig. 4B) compared with those obtained using parabolic flight (Newman 1996; Donelan and Kram 2000) show that the stride length with the vertical BWS is smaller than under real weightlessness. Therefore, our tilted BWS system (Fig. 1A, right panel) 
gives a better approximation of the swing phase dynamics under real weightlessness than does the vertical BWS system (Donelan and Kram 1997; 2000) or a simulator in a horizontal supine position using elastic cords for leg suspension (McCrory et al. 2002; De Witt et al. 2010).

Are these differences in the swing phase dynamics and limb segment motion negligible? To answer this question, one should likely consider compensatory effects and an interrelation between different gait parameters rather than a simple comparison of single parameters. Even though the maximal longitudinal foot velocity for the tilted BWS condition decreased only slightly relative to the vertical BWS system (Fig. 2B and 3B, bottom right panels), however, the actual decrement was much more obvious if one takes into account that it was significantly compensated for or masked by increments in the stride length. A previous modeling study also predicts differential effects of gravity during stance and swing phases (Raichlen 2008). In fact, the changes in the longitudinal foot excursion were basically opposite for the vertical and tilted BWS systems. For the former system the amplitude of longitudinal foot motion decreased, while for the latter system it increased relative to the $1 \mathrm{~g}$ condition when walking at the same speed (Fig. 2B). Considering a monotonic (presumably proportional, Fig. 6A) relationship between the stride length and the maximal foot velocity at a given gravity level (1g), the peak foot velocity would be expected to be 1.5 times higher for the vertical than for tilted BWS condition if the stride lengths were similar (Fig. 6B).

Overall, our results demonstrated that gravity acting on both stance and swing legs play an important role in shaping locomotor patterns. The stride length and the duration of the step cycle depend on the gravity load on the swinging leg (Fig. 2B,4B), so that the swing phase might have more influence on gait than it was previously thought. For instance, slower swing and longer foot excursions in the tilted BWS condition (Fig. 2,4) may raise questions about optimality and/or 'comfort' of walking, and could account for potentially different preferred gaits, such as loping on the Moon observed in Apollo astronauts (though the Lunar suit limits the range of motion in the leg joints and may also contribute to the loping gait on the Moon; Carr and McGee, 2009). Therefore, the results may support the idea of looking for new forms of locomotion in a heterogravity 
environment (Srinivasan and Ruina 2006; Minetti et al. 2012), based on the interplay between stance and swing phase dynamics, changes in the intersegmental coordination and their consequences on the COM motion. The present findings support the usage of BWS systems for approximating reduced gravity in the laboratory, and confirm the important role of the CNS in phase shift regulations for adjusting the basic patterns in altered locomotor conditions (Grillner 1981; Lacquaniti et al. 2002; Barliya et al. 2009; Maclellan and McFadyen 2010). 


\section{Acknowledgements}

The financial support of Italian Health Ministry, Italian University Ministry (PRIN project), Italian Space Agency (CRUSOE grant) and EU FP7-ICT program (MINDWALKER grant \#247959 and AMARSi grant \#248311) is gratefully acknowledged. 


\section{References}

Alexander RM (1989) Optimization and gaits in the locomotion of vertebrates. Physiol Rev 69: 1199-1227.

Barliya A, Omlor L, Giese MA, Flash T (2009) An analytical formulation of the law of intersegmental coordination during human locomotion. Exp Brain Res 193(3):371-85.

Bianchi L, Angelini D, Orani GP, Lacquaniti F (1998) Kinematic co-ordination in human gait: relation to mechanical energy cost. J Neurophysiol 79: 2155-2170.

Bogdanov VA, Gurfinkel VS, Panfilov VE (1971) Human motion under lunar gravity conditions. Kosm Biol Med (Space Biology and Medicine) 5:3-13, [russian].

Borghese NA, Bianchi L, Lacquaniti F (1996) Kinematic determinants of human locomotion. J Physiol 494: 863-879.

Browning RC, Modica JR, Kram R, Goswami A (2007) The effects of adding mass to the legs on the energetics and biomechanics of walking. Med Sci Sports Exerc 39: 515-525.

Cappellini G, Ivanenko YP, Dominici N, Poppele RE, Lacquaniti F (2010) Motor pool activity migration in the spinal cord reflects body mechanics in human locomotion. J Neurophysiol 104(6): 3064-3073.

Carr CE, McGee J (2009) The Apollo Number: space suits, self-support, and the walk-run transition. PLoS One 4(8):e6614.

Cavagna GA, Heglund NC, Taylor CR (1977) Mechanical work in terrestrial locomotion: two basic mechanisms for minimizing energy expenditure. The American journal of physiology 233(5): R243-261.

Cavagna GA, Willems PA, Heglund NC (2000) The role of gravity in human walking: pendular energy exchange, external work and optimal speed. J Physiol 528(Pt 3): 657-668.

Chang YH, Huang HW, Hamerski CM, Kram R (2000) The independent effects of gravity and inertia on running mechanics. J Exp Biol 203:229-238. 
Cheron G, Bouillot E, Dan B, Bengoetxea A, Draye JP, Lacquaniti F (2001) Development of a kinematic coordination pattern in toddler locomotion: planar covariation. Exp Brain Res 137: 455-466.

Courtine G, Roy RR, Hodgson J, McKay H, Raven J, Zhong H, Yang H, Tuszynski MH, Edgerton VR (2005) Kinematic and EMG determinants in quadrupedal locomotion of a non-human primate (Rhesus). J Neurophysiol 93: 3127-3145.

Courtine G, Schieppati M (2004) Tuning of a basic coordination pattern constructs straight-ahead and curved walking in humans. J Neurophysiol 91(4):1524-1535.

De Witt JK, Perusek GP, Lewandowski BE, Gilkey KM, Savina MC, Samorezov S, Edwards WB (2010) Locomotion in simulated and real microgravity: horizontal suspension vs. parabolic flight. Aviat Space Environ Med 81(12): 1092-1099.

Dietz V, Duysens J (2000) Significance of load receptor input during locomotion: a review. Gait Posture 11: 102-110.

Doke J, Donelan JM, Kuo AD (2005) Mechanics and energetics of swinging the human leg. J Exp Biol 208: 439-445.

Doke J, Kuo AD (2007) Energetic cost of producing cyclic muscle force, rather than work, to swing the human leg. J Exp Biol 210: 2390-2398.

Dominici N, Ivanenko YP, Lacquaniti F (2007) Control of foot trajectory in walking toddlers: adaptation to load changes. J Neurophysiol 97(4): 2790-2801.

Donelan JM, Kram R (1997) The effect of reduced gravity on the kinematics of human walking: a test of the dynamic similarity hypothesis for locomotion. J Exp Biol 200(Pt 24): 3193-3201.

Donelan JM, Kram R (2000) Exploring dynamic similarity in human running using simulated reduced gravity. J Exp Biol 203(Pt 16): 2405-2415.

Everett ME, O'Connor DP, Dewitt JK (2009) Lower limb position during treadmill jogging and fast running in microgravity. Aviat Space Environ Med 80(10): 882-886. 
Funato T, Aoi S, Oshima H, Tsuchiya K (2010) Variant and invariant patterns embedded in human locomotion through whole body kinematic coordination. Exp Brain Res 205(4):497-511.

Gosseye TP, Willems PA, Heglund NC (2010) Biomechanical analysis of running in weightlessness on a treadmill equipped with a subject loading system. Eur J Appl Physiol 110(4): 709-728.

Gravano S, Ivanenko YP, Maccioni G, Macellari V, Poppele RE, Lacquaniti F (2011) A novel approach to mechanical foot stimulation during human locomotion under body weight support. Hum Mov Sci 30(2):352-367.

Griffin TM, Tolani NA, Kram R (1999) Walking in simulated reduced gravity: mechanical energy fluctuations and exchange. J Appl Physiol 86(1): 383-390.

Grillner S (1981) Control of locomotion in bipeds, tetrapods, and fish. Handbook of Physiology. The Nervous System. Motor Control. Bethesda, MD: Am Physiol Soc 1(2):1179-1236.

Hallemans A, Aerts P (2009) Effects of visual deprivation on intra-limb coordination during walking in children and adults. Exp Brain Res 198(1):95-106.

Hansen JR (1995) Spaceflight revolution: NASA Langley Research Center from Sputnik to Apollo. NASA, Washington DC, NASA SP-4308.

Ivanenko YP, Cappellini G, Dominici N, Poppele RE, Lacquaniti F (2007) Modular control of limb movements during human locomotion. J Neurosci 27(41):11149-11161.

Ivanenko YP, Cappellini G, Poppele RE, Lacquaniti F (2008) Spatiotemporal organization of alphamotoneuron activity in the human spinal cord during different gaits and gait transitions. Eur $\mathrm{J}$ Neurosci 27(12): 3351-3368.

Ivanenko YP, Grasso R, Macellari V, Lacquaniti F (2002) Control of foot trajectory in human locomotion: role of ground contact forces in simulated reduced gravity. J Neurophysiol 87: 3070-3089.

Ivanenko YP, Sylos Labini F, Cappellini G, Macellari V, McIntyre J, Lacquaniti F (2011) Gait transitions in simulated reduced gravity. J Appl Physiol 110(3):781-788. 
Juvin L, Simmers J, Morin D (2007) Locomotor rhythmogenesis in the isolated rat spinal cord: a phase-coupled set of symmetrical flexion extension oscillators. J Physiol 583(Pt 1): 115-128.

Kram R, Domingo A, Ferris DP (1997) Effect of reduced gravity on the preferred walk-run transition speed. J Exp Biol 200(Pt 4): 821-826.

Kuo AD (2001) A simple model of bipedal walking predicts the preferred speed-step length relationship. J Biomech Eng 123: 264-269.

Kuo AD, Donelan JM, Ruina A (2005) Energetic consequences of walking like an inverted pendulum: step-to-step transitions. Exerc Sport Sci Rev 33: 88-97.

Lacquaniti F, Grasso R, Zago M (1999) Motor Patterns in Walking. News Physiol Sci 14:168-174.

Lacquaniti F, Ivanenko YP, Zago M (2002) Kinematic control of walking. Arch Ital Biol 140(4):263-272.

Leurs F, Bengoetxea A, Cebolla AM, De Saedeleer C, Dan B, Cheron G (2012) Planar covariation of elevation angles in prosthetic gait. Gait Posture 35(4):647-652.

MacLellan MJ, McFadyen BJ (2010) Segmental control for adaptive locomotor adjustments during obstacle clearance in healthy young adults. Exp Brain Res 202(2):307-318.

Margaria R (1976) Biomechanics and Energetics of Muscular Exercise. Oxford: Clarendon.

Marsh RL, Ellerby DJ, Carr JA, Henry HT, Buchanan CI (2004) Partitioning the energetics of walking and running: swinging the limbs is expensive. Science 303: 80-83.

McCrory JL, Baron HA, Balkin S, Cavanagh PR (2002) Locomotion in simulated microgravity: gravity replacement loads. Aviat Space Environ Med 73(7): 625-631.

Minetti AE (2001) Invariant aspects of human locomotion in different gravitational environments. Acta Astronaut 49(3-10): 191-198.

Minetti AE, Ivanenko YP, Cappellini G, Dominici N, Lacquaniti F (2012) Humans running in place on water at simulated reduced gravity. PLoS One 7(7):e37300.

Mochon S, McMahon TA (1980) Ballistic walking. J Biomech 13: 49-57.

Newman DJ (1996) Modeling reduced gravity human locomotion. Int J Appl Sci Comp 3: 91-101. 
Noble JW, Prentice SD (2008) Intersegmental coordination while walking up inclined surfaces: age and ramp angle effects. Exp Brain Res 189(2):249-255.

Ogihara N, Kikuchi T, Ishiguro Y, Makishima H, Nakatsukasa M (2012) Planar covariation of limb elevation angles during bipedal walking in the Japanese macaque. J R Soc Interface 9(74):21812190.

Osaki Y, Kunin M, Cohen B, Raphan T (2007) Three-dimensional kinematics and dynamics of the foot during walking: a model of central control mechanisms. Exp Brain Res 176(3): 476-496.

Osaki Y, Kunin M, Cohen B, Raphan T (2008) Relative contribution of walking velocity and stepping frequency to the neural control of locomotion. Exp Brain Res 185(1):121-135.

Raichlen DA (2008) The effects of gravity on human walking: a new test of the dynamic similarity hypothesis using a predictive model. J Exp Biol 211(Pt 17):2767-72.

Roberts JF (1963) Walking Responses under Lunar and Low Gravity Conditions: Wright-Patterson AFB, OH: Aerospace Medical Research Laboratory.

Saibene F, Minetti AE (2003) Biomechanical and physiological aspects of legged locomotion in humans. Eur J Appl Physiol 88: 297-316.

Selionov VA, Ivanenko YP, Solopova IA, Gurfinkel VS (2009) Tonic central and sensory stimuli facilitate involuntary air-stepping in humans. J Neurophysiol 101(6): 2847-2858.

Srinivasan M, Ruina A (2006) Computer optimization of a minimal biped model discovers walking and running. Nature 439, 72-75.

Sylos Labini F, Ivanenko YP, Cappellini G, Gravano S, Lacquaniti F (2011) Smooth changes in the EMG patterns during gait transitions under body weight unloading. J Neurophysiol 106(3):15251536.

White O, Bleyenheuft Y, Ronsse R, Smith AM, Thonnard JL, Lefèvre P (2008) Altered gravity highlights central pattern generator mechanisms. J Neurophysiol 100(5): 2819-2824. 


\section{Figure legends}

Fig. 1: Schematic diagrams of simulated reduced gravity systems. A - the vertical BWS system applying an upward vertical force $(F)$ on the torso of the participant (left) and the tilting BWS system which simulates reduced gravity through the vector $m g^{*} \cos ($ alpha). B - the simulation of gravity at $0.16 \mathrm{~g}$ using three reduced gravity simulations.

Fig. 2: Gait characteristics for simulated walking $(2 \mathrm{~km} / \mathrm{h})$ at $0.16 \mathrm{~g}$. A - temporal gait characteristics for the different experimental conditions. B - ensemble averaged ( $\mathrm{n}=7$ subjects) longitudinal foot velocity trajectories over the gait cycle across walking conditions and the corresponding phase-plane diagrams. The bar graphs illustrate changes in maximum longitudinal foot velocity and longitudinal foot excursion across conditions (mean \pm SD). For comparison, the data for walking at $1 \mathrm{~g}$ at $5 \mathrm{~km} / \mathrm{h}$ (corresponding to the matched dimensionless Froude number as 2 $\mathrm{km} / \mathrm{h}$ at $0.16 \mathrm{~g})$ are also depicted. Horizontal dashed lines indicate values for walking $(2 \mathrm{~km} / \mathrm{h})$ at $1 \mathrm{~g}$. Significant differences $(\mathrm{p}<0.05)$ from walking at $1 \mathrm{~g}$ at $2 \mathrm{~km} / \mathrm{h}$ are denoted by an *

Fig. 3: Intersegmental coordination during simulated walking $(2 \mathrm{~km} / \mathrm{h})$ at $0.16 \mathrm{~g}$. A - average trajectories for joint angular (hip, knee, ankle) and segment elevation (thigh, shank, foot) angles during the stride cycle for all walking and reduced gravity conditions. B - peak-to-peak joint angle and elevation angle amplitudes (mean $\pm S D, n=7$ subjects), $C$ - percent of variance accounted for by the 1st harmonic of each elevation angle and its phase angle (0 denotes the phase of cosine) and D the percent variance explained by the first (PV1) and second (PV2) principal components along with the orientation of the covariance plane as determined by the angle between the third principal component and thigh axis (u3t parameter). Horizontal dashed lines indicate values for walking (2 $\mathrm{km} / \mathrm{h})$ at $1 \mathrm{~g}$. Significant differences $(\mathrm{p}<0.05)$ from walking at $1 \mathrm{~g}$ at $2 \mathrm{~km} / \mathrm{h}$ are denoted by an *. 
Fig. 4: Gait characteristics for simulated running $(5 \mathrm{~km} / \mathrm{h})$ at $0.16 \mathrm{~g}$. For comparison, the data for running at a higher $(12 \mathrm{~km} / \mathrm{h})$ speed at $1 \mathrm{~g}$ are also depicted. The figure is in the same format as Fig. 2 and significant differences $(\mathrm{p}<0.05)$ from walking at $1 \mathrm{~g}$ at $5 \mathrm{~km} / \mathrm{h}$ are denoted by an *.

Fig. 5: Intersegmental coordination during simulated running ( $5 \mathrm{~km} / \mathrm{h})$ at $0.16 \mathrm{~g}$. The figure is in the same format as Fig. 3 and significant differences $(\mathrm{p}<0.05)$ from walking at $1 \mathrm{~g}$ at $5 \mathrm{~km} / \mathrm{h}$ are denoted by an *.

Fig. 6: Relationship between foot velocity and excursion during walking for different simulated gravity conditions. A - the results of the second-order model controlling the fore-aft position and velocity of the foot during walking at $1 \mathrm{~g}$ (adapted from Osaki et al. (2007) with permission). Different symbols correspond to different experimental conditions (natural walking at different speeds and with pacing cues (Osaki et al. 2007)). Note monotonic increments in the peak foot velocity with increasing in the foot excursion. B - changes in maximum longitudinal foot velocity and longitudinal foot excursion across conditions (same data as in Fig. 2B). Horizontal dashed lines indicate values for walking ( $2 \mathrm{~km} / \mathrm{h}$ ) at $1 \mathrm{~g}$. The bar with a diagonal pattern represents the maximal foot velocity value for the vertical BWS system approximated by matching the foot excursion to that of the tilted BWS system (indicated by arrows) based on a proportional relationship between the two parameters (see panel A). 
A

vertical BWS system

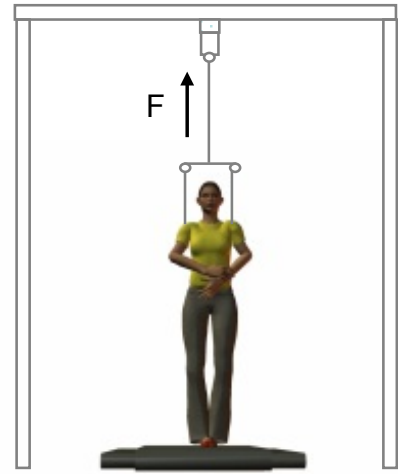

B simulated locomotion in reduced gravity $(0.16 \mathrm{~g})$

$F_{p}=0.16 \cdot m g$

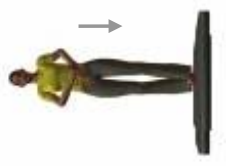

gravity replacement load tilted BWS system

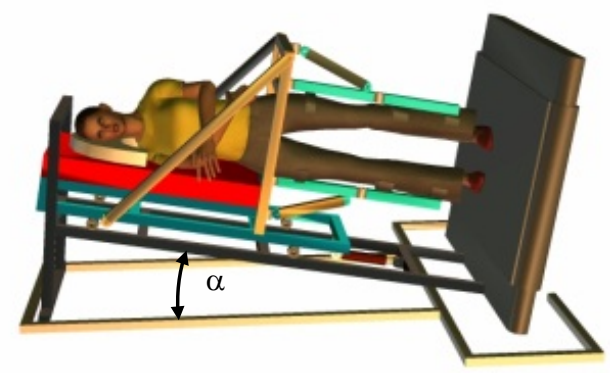

$F_{\text {vert }}=0.84 \cdot \mathrm{mg}$

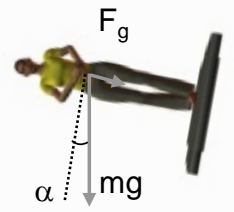

tilted BWS system

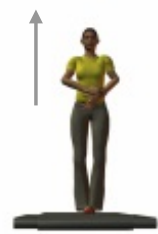

vertical BWS system

Fig. 1 
simulated walking at $0.16 \mathrm{~g}$

speed $=2 \mathrm{~km} / \mathrm{h}$

B
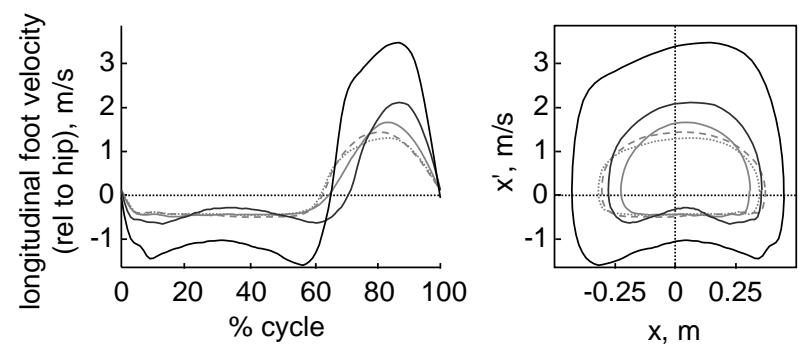

- gravity replacement load

$\square$ - tilted BWS system

$-1 \mathrm{~g}(2 \mathrm{~km} / \mathrm{h})$

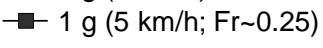

swing phase duration

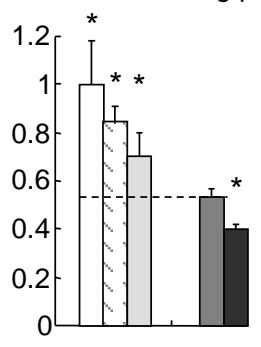

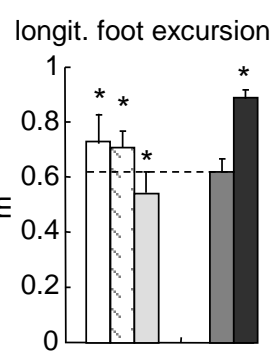

max longit. foot vel.

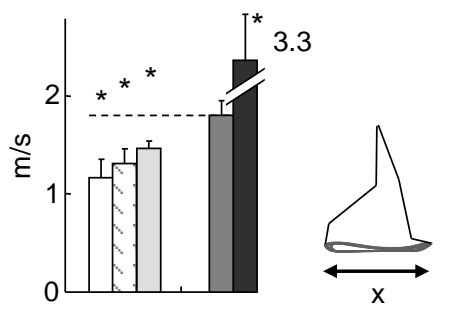

Fig. 2 
A

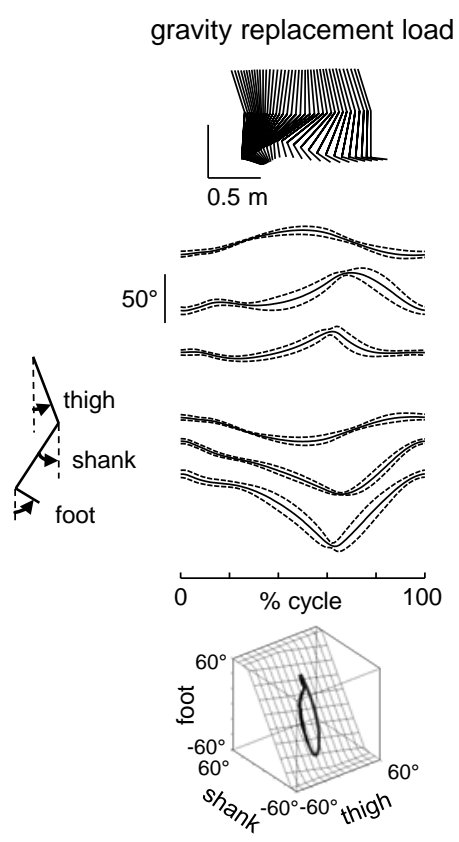

speed $=2 \mathrm{~km} / \mathrm{h}$

tilted BWS system
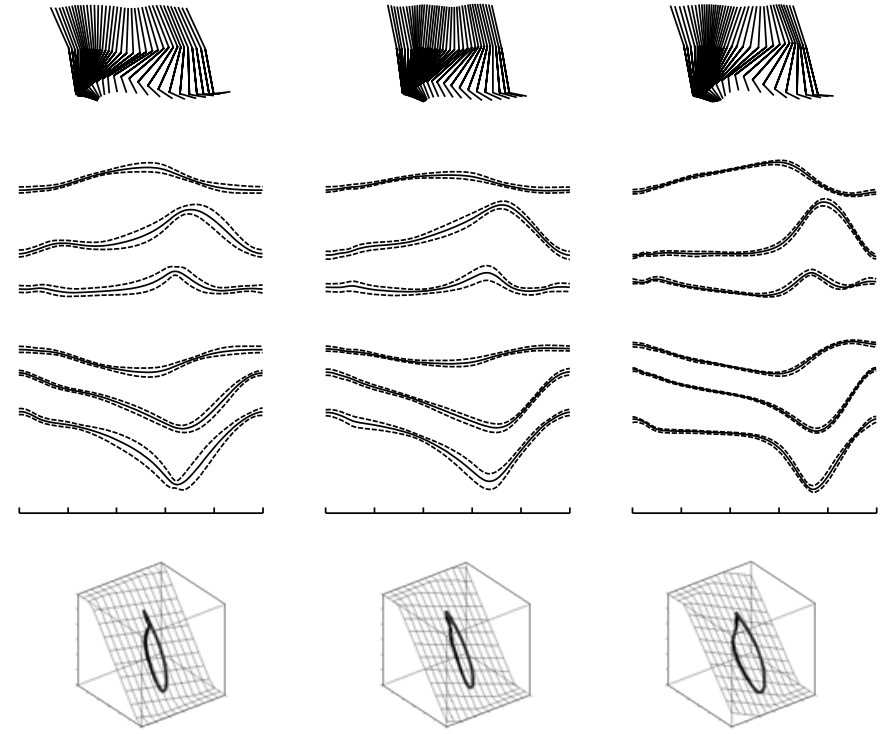

$1 \mathrm{~g}(2 \mathrm{~km} / \mathrm{h})$

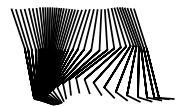

$1 \mathrm{~g}(5 \mathrm{~km} / \mathrm{h})$
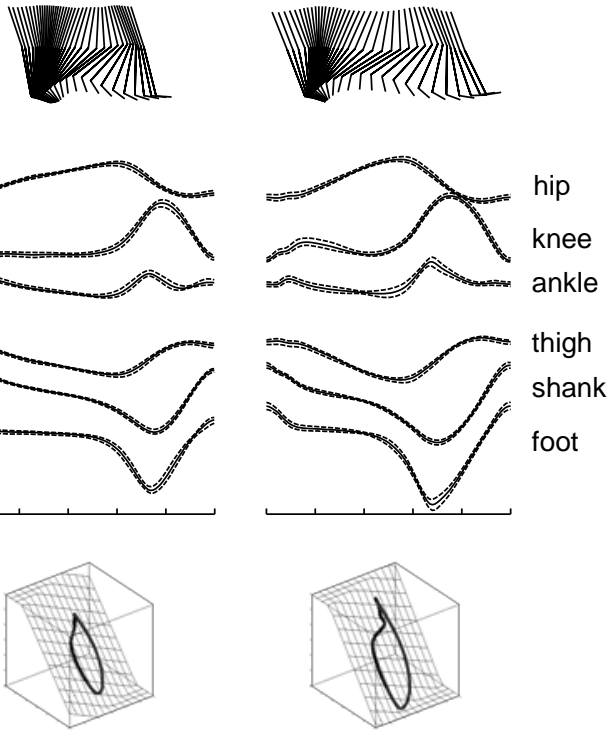

B
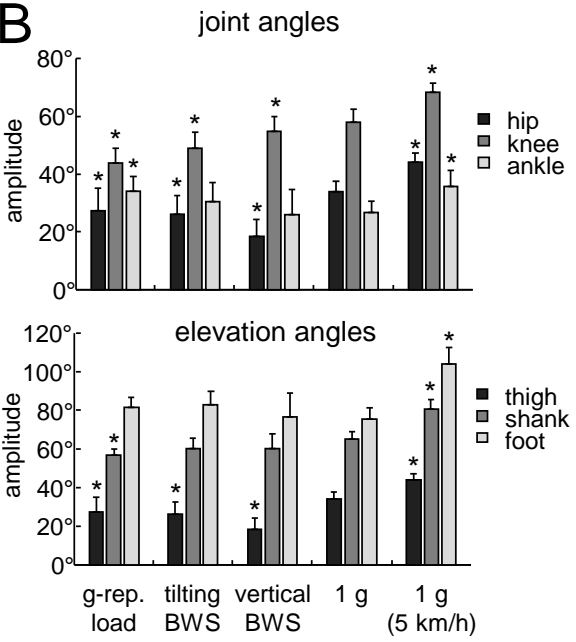

C
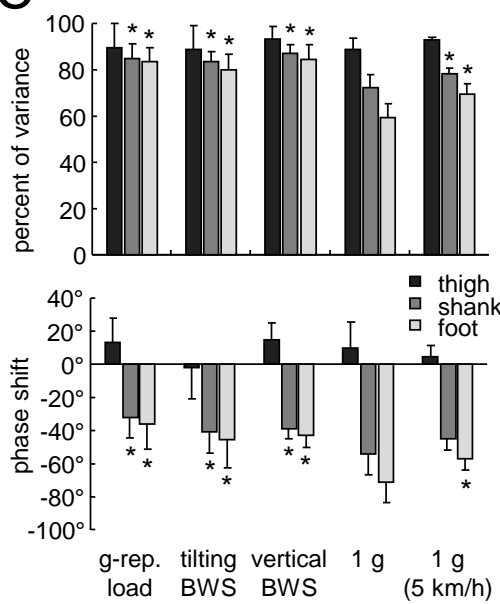

D planar covariance
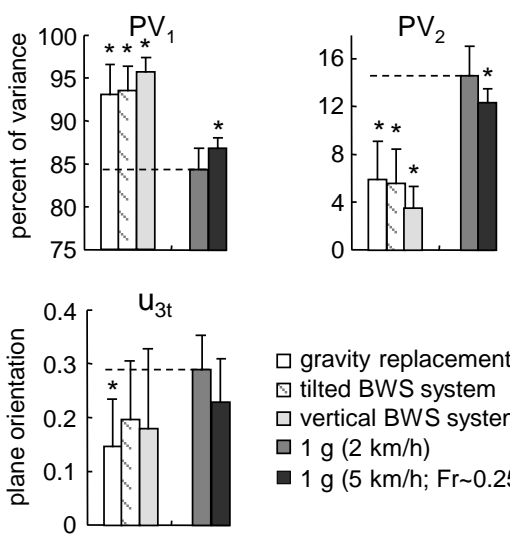

$\square$ gravity replacement load $\checkmark$ tilted BWS system $\square$ vertical BWS system $1 \mathrm{~g}(2 \mathrm{~km} / \mathrm{h})$

- 1 g (5 km/h; Fr 0.25) 
simulated running at $0.16 \mathrm{~g}$

A

cycle duration

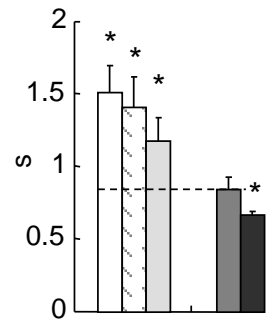

$\square$ gravity replacement load

- $\square$ - tilted BWS system

$\square$ - vertical BWS system

$\rightarrow-1 \mathrm{~g}(5 \mathrm{~km} / \mathrm{h})$

$\rightarrow-1 \mathrm{~g}(12 \mathrm{~km} / \mathrm{h})$

swing phase duration

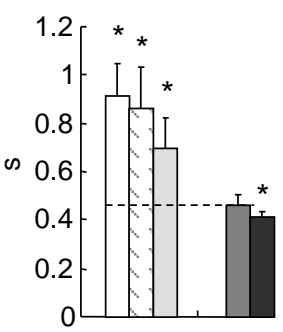

B
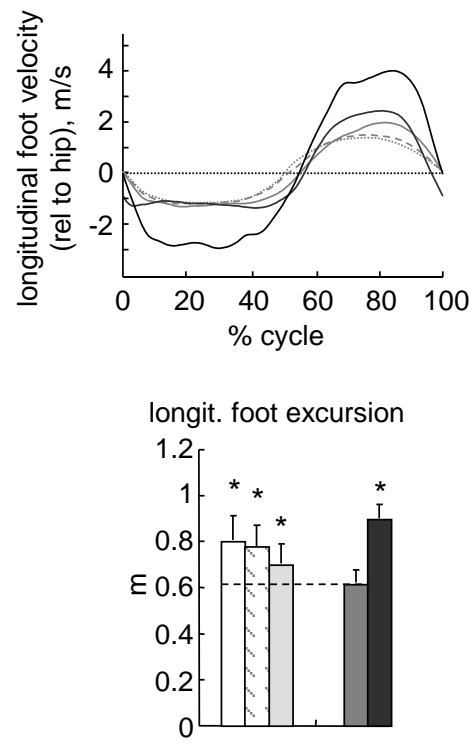

speed $=5 \mathrm{~km} / \mathrm{h}$

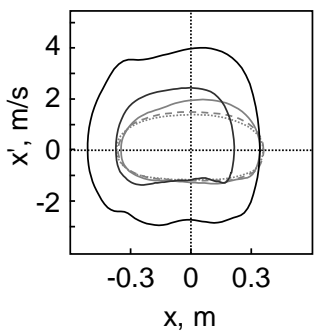

max longit. foot vel.

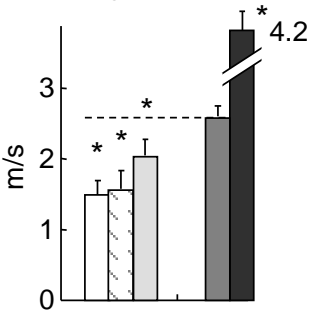

Fig. 4 
A

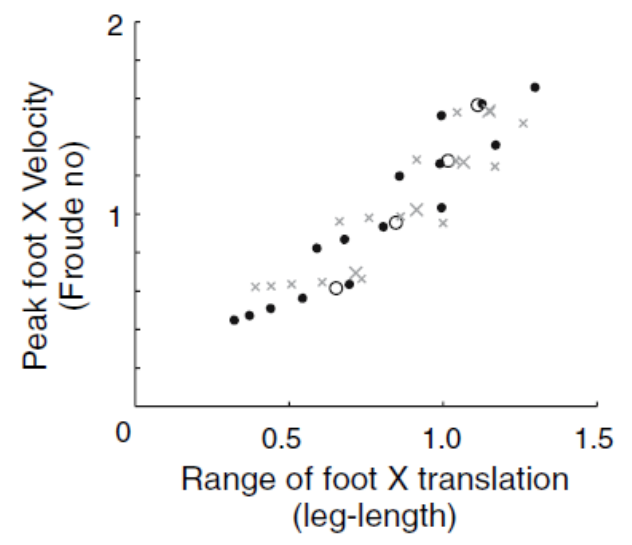

B

longit. foot excursion

max longit. foot vel.
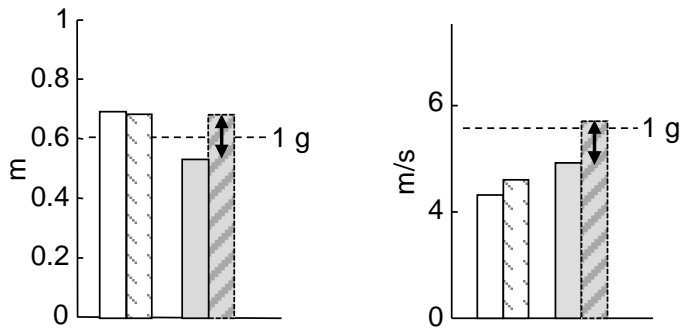

$\square$ gravity replacement load

$\square$ tilted BWS system

$\square$ vertical BWS system

$\square$ vertical BWS system (matched foot excursion)

Fig. 6 